\title{
Standard relativistic reference systems and the IAU framework
}

\author{
Michael Soffel \\ Lohrmann-observatory, TU Dresden \\ email: soffel@rcs.urz.tu-dresden.de
}

\begin{abstract}
The IAU framework for relativistic reference systems is based upon the work by Brumberg and Kopeikin and by Damour, Soffel and Xu (DSX). We begin with a brief introduction into the DSX-formalism. After that the various IAU Resolutions concerning relativistic astronomical reference systems are discussed. Finally, it is indicated how the expansion of the universe can be considered in the BCRS.
\end{abstract}

Keywords. Relativity, post-Newtonian approximation, astronomical reference systems.

\section{Introduction}

Soon after Einstein's seminal paper Einstein (1915) on General Relativity appeared it became obvious that solving Einstein's field equations for applications in the solar system becomes extremely complicated and one has to resort to approximation schemes. Consequently a slow-motion weak-field approximation called post-Newtonian approximation was worked out by Droste (1916), de Sitter (1916), Lorentz and Droste (1917) and then later by Fock (1959), Nordtvedt (1970) and Will (1993) who added a certain set of postNewtonian parameters allowing for a violation of Einstein's theory of gravity in nature at the corresponding level of approximation. This classical post-Newtonian framework (e.g., Will 1993), however, has a certain number of drawbacks. First, one single coordinate system is used e.g., for the description of the gravitational $N$ body problem. Second, matter is described as an ideal fluid (an exception can be found in Misner et al., 1973). And third, after the paper by Blanchet and Damour (1989) it became obvious that the classical post-Newtonian center-of-mass and mass definitions should be improved. With respect to the coordinate system problem, it is obvious that for the description of local physics in the vicinity of a body $A$ that is a member of some gravitational $N$-body problem, some local co-moving system, where the influence of external bodies is effaced and described in terms of tidal terms, should be employed.

\section{The DSX-framework}

In a series of papers Damour, Soffel and Xu (DSX) (Damour et al., 1991-1994; see also Brumberg and Kopeikin 1988) laid the foundations of a new and improved relativistic celestial mechanics describing a system of $N$ gravitationally interacting rotating bodies of arbitrary shape and composition at the first post-Newtonian approximation of Einstein's theory of gravity. No assumption is made on the internal composition of bodies. The basic matter variables of the DSX-formalism are the gravitational mass-energy

$$
\sigma \equiv \frac{T^{00}+T^{s s}}{c^{2}}
$$


where $T^{\mu \nu}$ are the components of the energy-momentum tensor and the gravitational mass-energy current density

$$
\sigma^{i} \equiv \frac{T^{0 i}}{c} .
$$

In the gravitational $N$-body problem the DSX-framework employs a total of $N+1$ different coordinate systems: one global system with coordinates $(c t, \mathbf{x})$ that covers the entire model manifold and one local $A$-system with coordinates $\left(c T_{A}, \mathbf{X}_{A}\right)$ that is comoving with body $A$ (Fig. 1). In each of the $N+1$ different coordinate systems the metric tensor is written in a special form. E.g., in the global system it is written as

$$
\begin{aligned}
g_{00} & =-1+\frac{2 w}{c^{2}}-\frac{2 w^{2}}{c^{4}}+\ldots, \\
g_{0 i} & =-\frac{4}{c^{3}} w^{i}+\ldots \\
g_{i j} & =\delta_{i j}\left(1+\frac{2 w}{c^{2}}\right)+\ldots
\end{aligned}
$$

Hence, the global metric tensor is completely determined by means of two potentials: a gravito-electric scalar potential $w$ and a gravito-magnetic vector potential $w^{i}$. The gravito-electric potential merely generalizes the usual Newtonian potential $U$; the vector potential $w^{i}$ describes the gravitational action of mass-energy currents (moving or rotating masses; Lense-Thirring effects). The corresponding Einstein field equations read:

$$
\begin{aligned}
\left(-\frac{1}{c^{2}} \frac{\partial^{2}}{\partial t^{2}}+\Delta\right) w & =-4 \pi G \sigma+\mathcal{O}\left(c^{-4}\right), \\
\Delta w^{i} & =-4 \pi G \sigma^{i}+\mathcal{O}\left(c^{-2}\right) .
\end{aligned}
$$

In the local $A$-system the metric is written in the same form but with potentials $W$ and $W^{a}$; there, the mass-energy density and corresponding current is written as $\Sigma$ and $\Sigma^{a}$.

The formalism employs a spatial set of post-Newtonian mass- and spin-multipole moments (Blanchet-Damour moments) that is used to skeletonize the metric potentials in

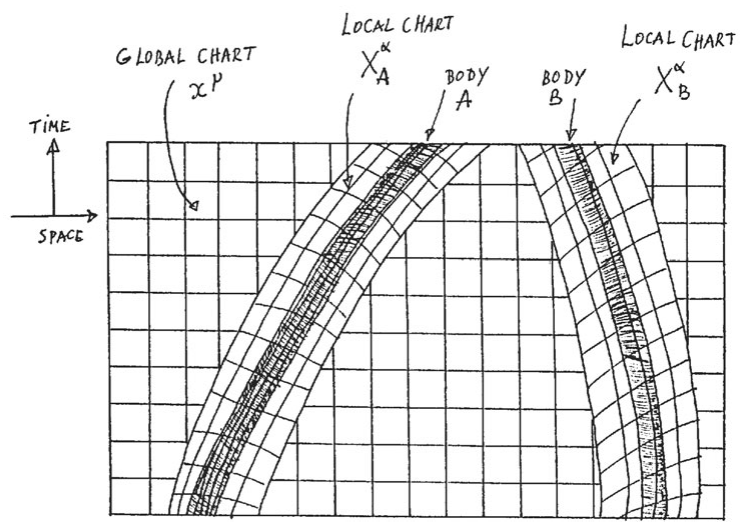

Figure 1. Various coordinate systems in the gravitational $N$-body system. 
the local $A$-system, generated by body $A$ itself outside of $A$ :

$$
\begin{aligned}
M_{L}^{A}(T) \equiv & \int_{A} d^{3} X \hat{X}^{L} \Sigma+\frac{1}{2(2 l+3) c^{2}} \frac{d^{2}}{d T^{2}} \int_{A} d^{3} X \hat{X}^{L} \mathbf{X}^{2} \Sigma \\
& -\frac{4(2 l+1)}{(l+1)(2 l+3) c^{2}} \frac{d}{d T} \int_{A} d^{3} X \hat{X}^{a L} \Sigma^{a} \quad(l \geqslant 0), \\
S_{L}^{A}(T) \equiv & \int_{A} d^{3} X \epsilon^{a b<c_{l}} \hat{X}^{L-1>a} \Sigma^{b} \quad(l \geqslant 1) .
\end{aligned}
$$

Here, the formalism of Cartesian STF- (symmetric and trace-free) tensors is used. The index $L$ is a multi-index standing for $l$ Cartesian indices: $L \equiv i_{1} i_{2} \ldots i_{l}$ with each Cartesian index $i=1,2,3=x, y, z$. The hat as well as the sharp brackets indicate that the corresponding symmetric and trace-free part (see e.g., Damour et al., 1991 for more details) has to be taken.

The heart of the DSX-formalism are the transformation rules for both the coordinates

$$
(c t, \mathbf{x}) \longleftrightarrow\left(c T_{A}, \mathbf{X}_{A}\right)
$$

and the metric potentials

$$
\left(w, w^{i}\right) \longleftrightarrow\left(W, W^{a}\right)_{A} .
$$

It is interesting to note that the Einstein-Infeld Hoffmann equations of motion that form the basis of any modern numerical ephemeris can simply be derived from

$$
W_{A}=\frac{G M_{A}}{R_{A}}
$$

and $W_{A}^{a}=0$ by a transformation of the metric in the local $A$-systems into the global (BCRS) system as geodetic equations of the BCRS metric tensor (Damour et al., 1991).

\section{Standard astronomical reference systems and IAU resolutions}

The IAU has recommended the use of two basic celestial reference systems: the Barycentric Celestial Reference System (BCRS) and the Geocentric Celestial Reference System (GCRS) (Soffel et al., 2003). Both systems are needed to replace the Newtonian concept of a quasi-inertial space fixed system. The BCRS with coordinates $(c t, \mathbf{x})$ is defined by the form of the metric tensor as in (2.1) together with the condition for asymptotic flatness:

$$
\lim _{r \rightarrow \infty}\left(w, w^{i}\right)=0
$$

This definition leaves the orientation of spatial BCRS axes open. Later this was fixed by the ICRF. IAU 2006 Resolution B2 (Default orientation of the Barycentric Celestial Reference System (BCRS) and Geocentric Celestial Reference System (GCRS) reads:

\section{.... Recommends}

"that the BCRS definition is completed with the following: "For all practical applications, unless otherwise stated, the BCRS is assumed to be oriented according to the ICRS axes. The orientation of the GCRS is derived from the ICRS-oriented BCRS.'

Note, that the BCRS is used for solar system ephemerides (dynamical equations of motion for solar system bodies), for problems of interplanetary spacecraft navigation, highprecision astrometry, for a definition of proper motion or radial velocity, etc. 
The GCRS was adopted by the IAU (2000) to model physical processes in the vicinity of the Earth. It is defined by the form of the metric tensor.

$$
\begin{aligned}
G_{00} & =-1+\frac{2 W}{c^{2}}-\frac{2 W^{2}}{c^{4}}+\ldots \\
G_{0 a} & =-\frac{4}{c^{3}} W^{a}+\ldots \\
G_{a b} & =\delta_{a b}\left(1+\frac{2 W}{c^{2}}\right)+\ldots
\end{aligned}
$$

In addition it is assumed that the spatial GCRS-coordinates are kinematically nonrotating with respect to the BCRS, i.e, they are locally non-inertial.

The quasi-linearity of the post-Newtonian field equations allows a unique split of the metric potentials $W$ and $W^{a}$ into three parts: i) internal, ii) inertial and iii) external parts. The internal parts result from the gravitational action of the Earth itself. In the absence of external bodies they present the post-Newtonian potentials of an isolated Earth, usually skeletonized by corresponding Blanchet-Damour moments. Outside the Earth these parts admit an expansion in terms of negative powers of $R$ (the coordinate distance to the geocenter). For practical applications the internal parts of $W$, the gravitational potentials of the Earth itself, can be written as an expansion that is given by IAU2000 Resolution B1.4 (see below). Terms of first order in $R$ are inertial terms. The corresponding term in $W$ describes a deviation from free-fall due to the oblateness of the Earth, the one in $W^{a}$ describes a Coriolis-force due to geodesic precession. A geocentric dynamically non-rotating system, where inertial forces are absent in dynamical equations of motion thus rotates with respect to the GCRS. The influence of external bodies is described as tidal terms, i.e., terms in the metric potentials that are at least quadratic in $R$. For more details the reader is referred to Soffel et al. (2003). The Geocentric Coordinate Time T is called TCG.

IAU 2000 Resolution B1.4, concerning the post-Newtonian expansion of the Earth's gravitational potentials reads:

The IAU .... Recommends

1. expansion of the post-Newtonian potential of the Earth in the Geocentric Celestial Reference System (GCRS) outside the Earth in the form

$$
W_{E}=\frac{G M_{E}}{R}\left[1+\sum_{l=2}^{\infty} \sum_{m=0}^{l}\left(\frac{R_{E}}{R}\right)^{l} P_{l m}(\cos \theta)\left(C_{l m}^{E}(T) \cos m \phi+S_{l m}^{E}(T) \sin m \phi\right],\right.
$$

where $C_{l m}^{E}$ and $S_{l m}^{E}$ are, to sufficient accuracy, equivalent to the post-Newtonian multipole moments introduced by Damour et al. (Damour et al., Phys. Rev. D, 43, 3273, 1991). $\theta$ and $\phi$ are the polar angles corresponding to the spatial coordinates $X^{a}$ of the GCRS and $R=|\mathbf{X}|$, and

2. expression of the vector potential outside the Earth, leading to the well-known LenseThirring effect, in terms of the Earth's total angular momentum vector $\mathbf{S}_{E}$ in the form

$$
W_{E}^{a}=-\frac{G}{2} \frac{\left(\mathbf{X} \times \mathbf{S}_{E}\right)^{a}}{R^{3}} .
$$

Though the expansion for $W_{E}$ looks completely Newtonian it is relativistic due to the use of BD-moments or their spherical equivalents $\left(C_{l m}^{E}, S_{l m}^{E}\right)$. That implies that the DSXformalism has been designed so that many relativistic terms are absorbed in the 
definitions of multipole moments. In practice they simply will be fit-parameters and a split into Newtonian- and post-Newtonian parts is superfluous, dangerous and confusing.

The GCRS is used to describe physical processes in the vicinity of the Earth, for the definitions of potentials coefficients (multipole moments), for satellite theory, for the description of dynamics of the Earth itself, for the definition of the CIP the CIO, etc, and for a direct relation to terrestrial systems and frames (ITRS and ITRF).

A set of auxiliary times scales has been linked to TCG and TCB. Originally Terrestrial Time was related with proper time of a clock located on the rotating geoid. However, IAU 2000 Resolution B1.9 (Re-definition of Terrestrial Time TT) recommends

that TT be a time scale differing from TCG by a constant rate: dTT/dTCG $=1-L_{G}$, where $L_{G}=6.969290134 \times 10^{-10}$ is a defining constant. International Atomic Time TAI is related with TT by

$$
\mathrm{TT}=\mathrm{TAI}+32.184 \mathrm{~s}
$$

For barycentric ephemerides the times scale TDB was introduced. IAU 2006 Resolution B3 recommends:

that, in situations calling for the use of a coordinate time scale that is linearly related to Barycentric Coordinate Time (TCB) for an extended time span, TDB be defined as the following linear transformation of TCB:

$$
\mathrm{TDB}=\mathrm{TCB}-L_{B} \times\left(\mathrm{JD}_{\mathrm{TCB}}-T_{0}\right) \times 86400+\mathrm{TDB}_{0}
$$

where $T_{0}=2443144.5003725$, and $L_{B}=1.550519768 \times 10^{-8}$ and $\mathrm{TDB}_{0}=-6.55 \times 10^{-5} \mathrm{~s}$ are defining constants. Note, that TDB was chosen to practically agree with the time argument of DE405.

\section{The BCRS-metric and the expansion of the universe}

So far the BCRS-metric was chosen to be asymptotically flat and all cosmological effects have been ignored, That is fine for the description of planetary motion and the propagation of light-rays in the solar system. However, at cosmological distances the expansion of the universe should be considered to deal with cosmological redshifts and the problem of various distance indicators (parallax-, luminosity-, angular diameter- and proper motion distance) that have to be distinguished for remote objects. Motivated also by the so-called Pioneer anomaly we included the expansion of the universe in the BCRSmetric and estimated orders of magnitude for corresponding effects in the solar system. Details can be found in Klioner and Soffel (2004). In the BCRS the expansion of the universe can be considered as a cosmic tidal force. The corresponding tidal acceleration grows with heliocentric distance; at Pluto's orbit it amounts to $2 \times 10^{-23} \mathrm{~m} / \mathrm{s}^{2}$ and points away from the Sun (Note, that the Pioneer anomaly amounts to $8.7 \times 10^{-10} \mathrm{~m} / \mathrm{s}^{2}$ and points towards the Sun). The perturbations of planetary orbits are completely negligible (the cosmic perihelion precession of Pluto's orbits is of order $10^{-5} \mu \mathrm{as} /$ century).

\section{References}

Blanchet, J. \& Damour, T. 1989, Annales de l'institut Henri Poincare (A) Physique theorique, 50, no. 4,377

Brumberg, V. \& Kopeikin, S. 1988, Nuovo Cimento B, 103, 63

Damour, T., Soffel, M., \& Xu, C. 1991, Phys. Rev. D, 43, 3273 (DSX I); 1992, 45, 1017 (DSX II); 1993, 47, 3124 (DSX III); 1994, 49, 618 (DSX IV)

de Sitter, W. 1916, Mon. Not. R. Astron. Soc., 76, 699 and 77, 155 
Droste, J. 1916, Versl. K. Akad. Wet. Amsterdam, 19, 447

Einstein, A. 1915, Preuss. Akad. Wiss. Berlin, Sitzber., 831

Fock, V. 1959 The Theory of Space, Time and Gravitation, Pergamon Press, Oxford

Klioner, S. \& Soffel, M., 2004, Refining the relativistic model for Gaia: cosmological effects in the BCRS, Proc. of the GAIA meeting 4-7 October 2004, Paris (ESA SP-576), 305-308

Lorentz, H.A. \& Droste, J., 1917 Versl. K. Akad. Wet. Amsterdam, 26, 392 (part I); 26, 649 (part II), English translation in H. A. Lorentz, Collected Papers, edited by P. Zeeman and A. D. Fokker (Nijhoff, The Hague, 1937), Vol. V, pp. 330-355

Misner, C., Thorne, K., \& Wheeler, J.A., 1972, Gravitation, Freeman

Nordtvedt, K., 1970, Astrophys. J., 161, 1059

Soffel, M., et al., 2003, Astron. J., 126, 2687

Will, C., 1993, Theory and Experiment in gravitational physics, Cambridge University Press, Cambridge 